\title{
Design transportation route scheme based on artificial intelligence algorithm and computer simulation
}

\author{
Yuchuan Huang \\ School of North China Electricity Power University, Hebei 071000, China \\ 708967537@qq.com
}

Keywords: Computer simulation, Dijkstra algorithm, Floyd algorithm, graph theory, mathematical modeling.

Abstract. This article adopts Computer simulation and graph theoretic algorithm to choose the most reasonable place to convey drugs. And this article use mathematical modeling and computer programming to solve the problem of transport drugs.

\section{Symbols and definitions}

\begin{tabular}{|c|c|c|c|c|c|c|}
\hline \multirow{11}{*}{ GUINEA } & & NZEREKORE & \multirow[b]{2}{*}{ LEBERIA } & 15 & $\begin{array}{c}\text { MONTSE } \\
\text { RRAD }\end{array}$ & \\
\hline & 3 & $\begin{array}{c}\text { LOLA } \\
\text { MACENTA }\end{array}$ & & 16 & $\begin{array}{l}\text { GRANG COPE } \\
\text { MOUNT }\end{array}$ & \\
\hline & 4 & KISSIDOUGO & \multirow{9}{*}{$\begin{array}{l}\text { SIERRA } \\
\text { LEONE }\end{array}$} & $\begin{array}{l}17 \\
18\end{array}$ & $\begin{array}{l}\text { KENEMA } \\
\text { BO }\end{array}$ & $\begin{array}{c}27 \\
\text { KAMBIA }\end{array}$ \\
\hline & 5 & FARANAH & & 19 & MOYAMBA & \\
\hline & 6 & SIGUIRI & & 20 & KONO & \\
\hline & $\begin{array}{l}7 \\
8\end{array}$ & $\begin{array}{l}\text { TOUGUE } \\
\text { MALI }\end{array}$ & & 21 & KOINADUGU & \\
\hline & $\begin{array}{l}8 \\
9\end{array}$ & $\begin{array}{l}\text { MALI } \\
\text { BOFFA }\end{array}$ & & 22 & BOMBALI & \\
\hline & 10 & DUBREKA & & 23 & TONKOLILI & \\
\hline & $\begin{array}{l}11 \\
12\end{array}$ & $\begin{array}{l}\text { KINDIA } \\
\text { COYAH }\end{array}$ & & 24 & FREETOWN & \\
\hline & 13 & CONAKRY & & 25 & $\begin{array}{l}\text { WESTERN } \\
\text { RURAL }\end{array}$ & \\
\hline & 14 & FORECA & & 26 & PORTLOK & \\
\hline
\end{tabular}

\section{The foundation of model and computer simulation and calculate}

I have got the data of two cities in the three countries. However, the transport of the drugs is too complex to find the optimal scheme, so I find the best transport by mathematical modeling and computer simulation.

As the picture shows, considering the problem of national border, the three countries are respected into four parts Guinea is divided into two parts i.e. 1and 2, Liberia is 4 and Sierra Lenoe is 3. The round in the picture are signed 1to 27.To find the optimal scheme, we take the part 1 as an example . regarding Part 1 as a matrix $A=\left(a_{i j}\right)$. $a_{i j}$ means the distance between province $i$ and $j$. 


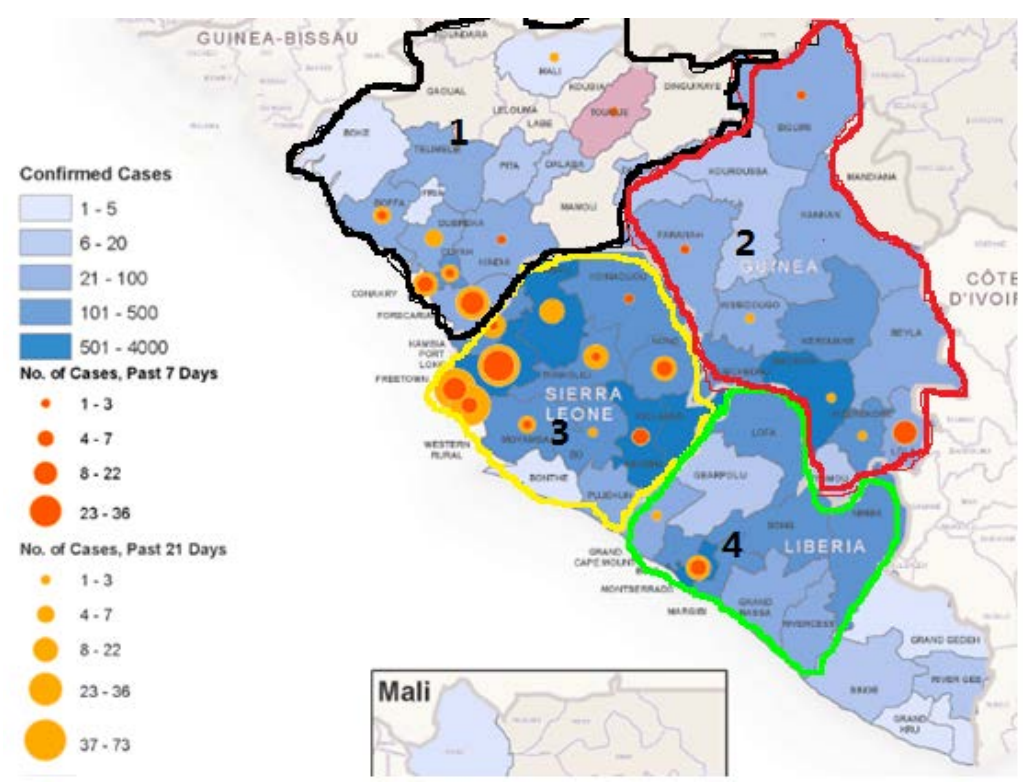

Figure 1

\section{Part 1}

Table 1

\begin{tabular}{|c|c|c|c|c|c|c|c|c|c|}
\hline & 7 & 8 & 9 & 10 & 11 & 12 & 13 & 14 & ST \\
\hline 7 & 0 & 0.7666 & 2.569 & 2.4184 & 1.7587 & 2.3838 & 2.7357 & 2.4192 & 15.0514 \\
\hline 8 & 0.7666 & 0 & 2.5609 & 2.6023 & 2.0767 & 2.6187 & 2.9241 & 2.7613 & 16.3106 \\
\hline 9 & 2.569 & 2.5609 & 0 & 0.6421 & 1.1659 & 0.8141 & 0.7447 & 1.2072 & 9.7039 \\
\hline 10 & 2.4184 & 2.6023 & 0.6421 & 0 & 0.725 & 0.1731 & 0.3231 & 0.5651 & 7.4491 \\
\hline 11 & 1.7587 & 2.0767 & 1.1659 & 0.725 & 0 & 0.6439 & 1.0132 & 0.6852 & 8.0686 \\
\hline 12 & 2.3838 & 2.6187 & 0.8141 & 0.1731 & 0.6439 & 0 & 0.3719 & 0.3939 & 7.3994 \\
\hline 13 & 2.7357 & 2.9241 & 0.7447 & 0.3231 & 1.0132 & 0.3719 & 0 & 0.6266 & 8.7393 \\
\hline 14 & 2.4192 & 2.7613 & 1.2072 & 0.5651 & 0.6852 & 0.3939 & 0.6266 & 0 & 8.6585 \\
\hline
\end{tabular}

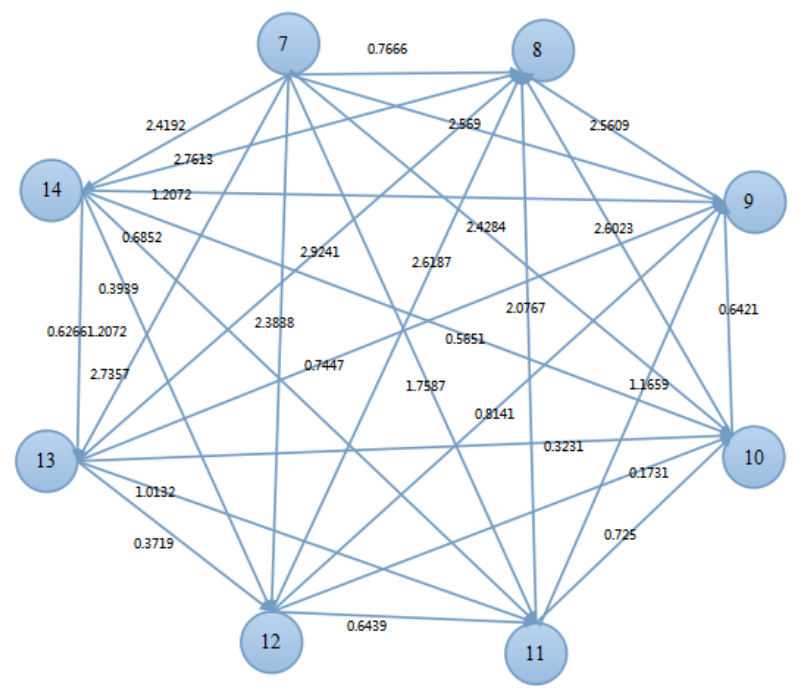

Figure 2

The distance of the 8 city points we abstract in the Part 1 are showed in Figure. After we calculate the shortest distance by Floyd, we can get a chart (Table), in which the summation of each row is the final result we need. Finally, we can get one of the best transport - COYAH! So, what is the concept 
about the best dispatch station? Actually it's a transfer station. It must have the minimum distance with the other points in the same part. In assumptions, the velocity of transport is constant, so the minimum distance means the shortest time. When the disease gets worse, we can delivery our main source to the best dispatch station that we have selected firstly, then we deliver the drug to the other region in the same part and spend a shortest time!

\section{4. $\quad$ Part 2 and Part 3}

\begin{tabular}{|c|c|c|c|c|c|c|c|}
\hline & 1 & 2 & 3 & 4 & 5 & 6 & ST \\
\hline 1 & 0 & 0.8422 & 1.0111 & 1.895 & 2.1976 & 3.6862 & 9.6321 \\
\hline 2 & 0.8422 & 0 & 1.6773 & 2.4024 & 1.3554 & 3.4308 & 9.7081 \\
\hline 3 & 1.0111 & 1.6773 & 0 & 0.8857 & 1.9267 & 2.9051 & 8.4059 \\
\hline 4 & 1.895 & 2.4024 & 0.8857 & 0 & 1.047 & 2.4382 & 8.6683 \\
\hline 5 & 2.1976 & 1.3554 & 1.9267 & 1.047 & 0 & 2.0754 & 8.6021 \\
\hline 6 & 3.6862 & 3.4308 & 2.9051 & 4.3822 & 2.0754 & 0 & 14.5357 \\
\hline
\end{tabular}

Part 2

\begin{tabular}{|c|c|c|c|c|c|c|c|c|c|c|c|c|}
\hline & 27 & 22 & 21 & 20 & 23 & 26 & 24 & 25 & 19 & 18 & 17 & ST \\
\hline $\begin{array}{l}2 \\
7 \\
\end{array}$ & 0 & $\begin{array}{c}0.768 \\
1 \\
\end{array}$ & $\begin{array}{c}1.216 \\
5 \\
\end{array}$ & $\begin{array}{c}2.048 \\
9 \\
\end{array}$ & $\begin{array}{c}1.135 \\
8 \\
\end{array}$ & $\begin{array}{c}0.445 \\
4\end{array}$ & $\begin{array}{c}0.709 \\
5 \\
\end{array}$ & $\begin{array}{c}0.827 \\
2 \\
\end{array}$ & $\begin{array}{c}1.088 \\
7 \\
\end{array}$ & $\begin{array}{c}1.659 \\
4\end{array}$ & $\begin{array}{c}2.138 \\
8 \\
\end{array}$ & $\begin{array}{c}12.0 \\
4 \\
\end{array}$ \\
\hline $\begin{array}{l}2 \\
2\end{array}$ & $\begin{array}{c}0.768 \\
1\end{array}$ & 0 & $\begin{array}{c}0.448 \\
4\end{array}$ & $\begin{array}{c}1.343 \\
1\end{array}$ & $\begin{array}{c}0.448 \\
4\end{array}$ & 1.052 & $\begin{array}{c}1.304 \\
3\end{array}$ & $\begin{array}{c}1.273 \\
3\end{array}$ & $\begin{array}{c}1.112 \\
5\end{array}$ & $\begin{array}{c}1.345 \\
7\end{array}$ & $\begin{array}{c}1.680 \\
6\end{array}$ & $\begin{array}{c}10.7 \\
8\end{array}$ \\
\hline $\begin{array}{l}2 \\
1\end{array}$ & $\begin{array}{c}1.216 \\
5\end{array}$ & $\begin{array}{c}0.448 \\
4\end{array}$ & 0 & 0.899 & $\begin{array}{c}0.694 \\
7\end{array}$ & $\begin{array}{c}1.500 \\
4\end{array}$ & $\begin{array}{c}1.752 \\
7\end{array}$ & $\begin{array}{c}1.721 \\
7\end{array}$ & $\begin{array}{c}1.560 \\
9\end{array}$ & $\begin{array}{c}1.614 \\
3\end{array}$ & $\begin{array}{c}1.678 \\
4\end{array}$ & $\begin{array}{c}13.0 \\
9\end{array}$ \\
\hline $\begin{array}{l}2 \\
0\end{array}$ & $\begin{array}{c}2.048 \\
9\end{array}$ & $\begin{array}{c}1.343 \\
1\end{array}$ & 0.899 & 0 & $\begin{array}{c}0.916 \\
1\end{array}$ & $\begin{array}{c}2.165 \\
2\end{array}$ & $\begin{array}{c}2.339 \\
1\end{array}$ & $\begin{array}{c}2.173 \\
3\end{array}$ & $\begin{array}{c}1.637 \\
2\end{array}$ & $\begin{array}{c}1.160 \\
7\end{array}$ & $\begin{array}{c}0.947 \\
7\end{array}$ & $\begin{array}{c}15.6 \\
3\end{array}$ \\
\hline $\begin{array}{l}2 \\
3\end{array}$ & $\begin{array}{c}1.135 \\
8\end{array}$ & $\begin{array}{c}0.448 \\
4\end{array}$ & $\begin{array}{c}0.694 \\
7\end{array}$ & $\begin{array}{c}0.916 \\
1\end{array}$ & 0 & 1.303 & $\begin{array}{c}1.514 \\
7\end{array}$ & $\begin{array}{c}1.405 \\
8\end{array}$ & $\begin{array}{c}1.035 \\
8\end{array}$ & $\begin{array}{c}1.021 \\
2\end{array}$ & $\begin{array}{c}1.267 \\
6\end{array}$ & $\begin{array}{c}10.7 \\
4 \\
\end{array}$ \\
\hline $\begin{array}{l}2 \\
6\end{array}$ & $\begin{array}{c}0.445 \\
4\end{array}$ & 1.052 & $\begin{array}{c}1.500 \\
4\end{array}$ & $\begin{array}{c}2.165 \\
2\end{array}$ & 1.303 & 0 & 0.271 & 0.402 & $\begin{array}{c}0.846 \\
1\end{array}$ & $\begin{array}{c}1.523 \\
3\end{array}$ & $\begin{array}{c}2.054 \\
4\end{array}$ & $\begin{array}{c}11.5 \\
6\end{array}$ \\
\hline $\begin{array}{l}2 \\
4 \\
\end{array}$ & $\begin{array}{c}0.709 \\
5 \\
\end{array}$ & $\begin{array}{c}1.304 \\
3 \\
\end{array}$ & $\begin{array}{c}1.752 \\
7 \\
\end{array}$ & $\begin{array}{c}2.339 \\
1 \\
\end{array}$ & $\begin{array}{c}1.514 \\
7\end{array}$ & 0.271 & 0 & $\begin{array}{c}0.267 \\
5 \\
\end{array}$ & $\begin{array}{c}0.866 \\
8 \\
\end{array}$ & $\begin{array}{c}1.576 \\
4\end{array}$ & $\begin{array}{c}2.124 \\
8 \\
\end{array}$ & $\begin{array}{c}12.7 \\
3 \\
\end{array}$ \\
\hline $\begin{array}{l}2 \\
5 \\
\end{array}$ & $\begin{array}{c}0.827 \\
2 \\
\end{array}$ & $\begin{array}{c}1.273 \\
3 \\
\end{array}$ & $\begin{array}{c}1.721 \\
7 \\
\end{array}$ & $\begin{array}{c}2.173 \\
3 \\
\end{array}$ & $\begin{array}{c}1.405 \\
8 \\
\end{array}$ & 0.402 & $\begin{array}{c}0.267 \\
5 \\
\end{array}$ & 0 & $\begin{array}{c}0.622 \\
1 \\
\end{array}$ & $\begin{array}{c}1.333 \\
7 \\
\end{array}$ & $\begin{array}{c}1.886 \\
1 \\
\end{array}$ & $\begin{array}{c}11.9 \\
1 \\
\end{array}$ \\
\hline $\begin{array}{l}1 \\
9 \\
\end{array}$ & $\begin{array}{c}1.088 \\
7 \\
\end{array}$ & $\begin{array}{c}1.112 \\
5\end{array}$ & $\begin{array}{c}1.560 \\
9\end{array}$ & $\begin{array}{c}1.637 \\
2\end{array}$ & $\begin{array}{c}1.035 \\
8\end{array}$ & $\begin{array}{c}0.846 \\
1\end{array}$ & $\begin{array}{c}0.866 \\
8\end{array}$ & $\begin{array}{c}0.622 \\
1\end{array}$ & 0 & $\begin{array}{c}0.711 \\
9\end{array}$ & 1.264 & $\begin{array}{c}10.7 \\
5 \\
\end{array}$ \\
\hline $\begin{array}{l}1 \\
8\end{array}$ & $\begin{array}{c}1.659 \\
4\end{array}$ & $\begin{array}{c}1.345 \\
7\end{array}$ & $\begin{array}{c}1.614 \\
3\end{array}$ & $\begin{array}{c}1.160 \\
7\end{array}$ & $\begin{array}{c}1.021 \\
2\end{array}$ & $\begin{array}{c}1.523 \\
3\end{array}$ & $\begin{array}{c}1.576 \\
4\end{array}$ & $\begin{array}{c}1.333 \\
7\end{array}$ & $\begin{array}{c}0.711 \\
9\end{array}$ & 0 & $\begin{array}{c}0.553 \\
9\end{array}$ & 12.5 \\
\hline $\begin{array}{l}1 \\
7\end{array}$ & $\begin{array}{c}2.138 \\
8\end{array}$ & $\begin{array}{c}1.680 \\
6\end{array}$ & $\begin{array}{c}1.678 \\
4\end{array}$ & $\begin{array}{c}0.947 \\
7\end{array}$ & $\begin{array}{c}1.267 \\
6\end{array}$ & $\begin{array}{c}2.054 \\
4\end{array}$ & $\begin{array}{c}2.124 \\
8\end{array}$ & $\begin{array}{c}1.886 \\
1\end{array}$ & 1.264 & $\begin{array}{c}0.553 \\
9\end{array}$ & 0 & 15.6 \\
\hline
\end{tabular}

Part 3

\section{Conclusion}

Finally I get four major transfer station,they are COYAH, MACENTA, TONKOLILI and GRANG COPE MOUNT. 


\section{Reference}

[1]The 65th WMA General Assembly, Durban, South Africa, October 2014.

[2]Y H Li,S P Chen.Evolutionary history of Ebola virus.[J].Epidemiology and Infection, 2014, 142(6):1138-1145.

[3]Marzi,A., Feldmann,H..Ebola virus vaccines: An overview of current approaches [J].Expert review of vaccines, 2014, 13(4):521-531.

[4]http://apps.who.int/ebola/en/ebola-situation-report/situation-reports/ebola-situation-report-4-febr uary-2015

[5]http://baike.haosou.com/doc/2458294.html

[6]Yunhui Zhang, Shu Wang, Yuqi Chen, Research progress of Ebora and 2014 situation of hemorrhagic fever with [J]. Infectious disease information,2014,(4):III-VIII.

[7]http://translate.google.com.hk/translate?hl=zh-CN\&sl=en\&u=http://en.wikipedia.org/wiki/Comp rehensive_National_Power\&prev=search

[8]http://translate.google.com.hk/translate?hl=zh-CN\&sl=en\&u=http://www.cnn.com/2014/08/04/h ealth/ebola-drug-questions/\&prev=search 